\title{
Valores de $\beta$-hidroxibutirato, ácidos graxos não esterificados, cortisol e insulina de cabras leiteiras com toxemia da prenhez subclínica durante o período de transição
}

Rodolfo José Cavalcanti Souto[a]" Gliére Silmara Leite Soares ${ }^{[b]}$, Alexandre Tadeu Mota Macedo $0^{[c]}$, Jobson Filipe de Paula Cajueiro $^{[a]}$,Daniel Nunes de Araújo Gonçalves ${ }^{[d]}$,Pierre Castro Soares ${ }^{[]]}, J o s e ́$ Augusto Bastos Afonso $0^{[a]}$, Carla Lopes de Mendonça $a^{[a]}$

\footnotetext{
[a] Clínica de Bovinos, Universidade Federal Rural de Pernambuco (UFRPE), Garanhuns, PE, Brasil

[b] Programa de Pós-Graduação em Sanidade e Reprodução de Ruminantes, Universidade Federal Rural de Pernambuco (UFRPE), Garanhuns, PE, Brasil

[c] Universidade do Vale do São Francisco (Univasf), Petrolina, PE, Brasil

[d] Programa de Pós-Graduação em Ciência Animal Tropical, Universidade Federal Rural de Pernambuco (UFRPE), Garanhuns, PE, Brasil

[e] Departamento de Medicina Veterinária, Universidade Federal Rural de Pernambuco (UFRPE), Garanhuns, PE, Brasil
}

*Autor correspondente

e-mail: rodolfojcsouto@gmail.com

\section{Resumo}

A toxemia da prenhez (T.P) ocorre na forma subclínica, sendo caracterizada pela elevação dos níveis de corpos cetônicos no sangue sem as manifestações clínicas da doença durante o período de transição. Este trabalho teve por objetivo avaliar as concentrações do $\beta$-hidroxibutirato, ácidos graxos não esterificados, cortisol e insulina de cabras leiteiras com T.P subclínica durante o período de transição. Foram acompanhadas 79 cabras leiteiras de alta produção, pluríparas, multíparas, mestiças e criadas em regime intensivo, em diferentes propriedades rurais. As cabras que apresentaram valores de $\mathrm{BHB} \leq 0,8 \mathrm{mmol} / \mathrm{L}$ em todos os momentos de avaliaçao ( $\mathrm{N}=40$ ) compuseram o grupo controle (G1). Foram consideradas como portadoras da T.P subclínica (G2) as cabras em que pelo menos um dos momentos experimentais apresentou valores de BHB entre $0,8 \mathrm{mmol} / \mathrm{L}$ a $1,6 \mathrm{mmol} / \mathrm{L}(\mathrm{N}=39)$. Os animais foram avaliados clínica e laboratorialmente 30 , 20, 10 dias antes do parto (dap), momento do parto e, 10, 20 e 30 dias pós-parto (dpp). Foram mensurados $\beta$-hidroxibutirato (BHB), ácidos graxos não-esterificados (AGNEs), cortisol e insulina. Foi utilizada a análise de variância (Teste F) com a finalidade de averiguar os efeitos e interações entre grupos e momentos, empregando-se programa computacional SAS. A maior ocorrência da T.P subclínica foi constatada durante o período de lactação. O BHB do G2 apresentou elevação significativa no momento do parto, mantendo valores 
superiores durante a lactação, quando comparado ao final da gestação. Os AGNE's do G2 foram superiores $(\mathrm{P}<0,01)$ ao $\mathrm{G} 1$, mantendo valores crescentes de concentração até o momento do parto, no qual atingiu valor médio máximo $(0,62 \mathrm{mmol} / \mathrm{l})$. Quanto à insulina, o G2 apresentou concentrações inferiores $(\mathrm{P}<0,01)$ em relação ao G1, enquanto o cortisol do G2 foi superior $(\mathrm{P}<0,05)$ ao do $\mathrm{G} 1$ ao longo de todos os momentos. A elevação do BHB se dá em função do aumento da demanda energética em virtude do crescimento fetal e produção de leite, estando mais elevado no início da lactação em virtude da dieta não atender a maior demanda de energia nessa fase. O aumento gradativo dos AGNE's com elevação no momento do parto acontece principalmente em função do envolvimento do sistema endócrino, pela a ação de hormônios lipolíticos. A redução mais expressiva na concentração de insulina no G2 é resultado do efeito deletério do AGNE's sobre a síntese deste hormônio. A elevação na concentração do cortisol, mais evidente no G2, está associada à condição de estresse resultante do transtorno metabólico. Desse modo, a T.P na forma subclínica está presente durante o período de transição, com maior ocorrência no período de lactação, desencadeando alterações marcantes nas concentrações séricas dos indicadores bioquímicos estudados. 